\section{Pan-Cancer Screening: A Dream or a Nightmare}

$\mathbf{I}$ $t$ stands to reason that the earlier you can diagnose cancer, the better chance you have to cure it. This idea has certainly held up in some diseases, such as those in which screening has clear value, like colorectal and lung cancers. However, for some cancers, such as prostate cancer, early diagnosis sometimes just leads to "watchful waiting," with continued surveillance and repeated biopsies until a clear need to treat is found.

This variability within and among cancers makes me concerned about new attempts to launch "pan-cancer" screening tests. On the surface, "one-stop shopping" seems good. And the test is easy. Forget surveillance radiology or endoscopy and physical examinations. Just a bit of blood, please.

But what happens when during your annual checkup a simple blood test is performed and results come back positive for cancer? Now what? First, we need to look for the cancer, but where should we start? Should we look at risk factors like smoking, and start there? Maybe we should just rule out more common cancers first. Or maybe we look for the most lethal cancers first? What kind of decision tree do we use to avoid unnecessary and sometimes risky procedures and make this an efficient process?

Also, don't forget that if these tests are very sensitive, we might be looking for a tumor so small that it falls below the level of detection of even the most advanced imaging modalities. What happens in that case? Do we just tell you that if it's that small, it's probably safe to wait and look again in a year? And now do you need a psychiatrist to deal with your anxiety?

I know that scenario is oversimplified and perhaps a little ridiculous, but hopefully you get my point. If we can move toward a pan-cancer test, I think we also need a second tier of blood-based testing to narrow the possibilities, ideally to just one organ, before we wade into a full-blown cancer-finding exercise. I do recognize that having universal blood-based screening that allows for avoiding traditional screening modalities would be cheaper and would ease the strain on the healthcare system. Although this still doesn't solve the problem of tiny cancers that defy radiologic imaging, it would spawn renewed interest in research with respect to molecular and functional imaging where, increasingly, subcentimeter lesions can be identified. This research is particularly important for organs that can be difficult to image, such as the pancreas for pancreatic adenocarcinoma, or for highly lethal cancers, such as glioblastoma multiforme.

To sum up, I do think pan-cancer screening could be a game changer. But we must remember the caveats, and approach this in a proper and orderly way to avoid doing more harm than good. JNCCN@nccn.org or log into www.editorialmanager.com/JNCCN.

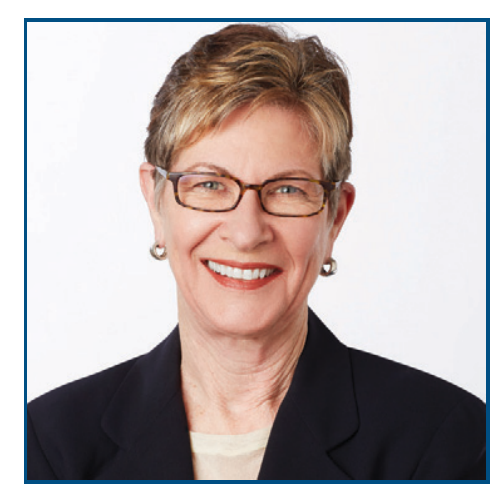

MARGARET TEMPERO, MD

Margaret Tempero, MD, is a Professor of Medicine and Director of the UCSF Pancreas Center and editor-in-chief of JNCCN. Her research career has focused on pancreatic ductal adenocarcinoma, especially in the area of investigational therapeutics. Dr. Tempero has served on the ASCO Board of Directors and as ASCO President. She currently serves on the ASCO Conquer Cancer Foundation Board. She codirected the AACR/ASCO Methods in Clinical Cancer Research and taught this course and similar courses in Europe and Australia. She was founding Chair of the $\mathrm{NCl}$ Clinical Oncology Study Section and served as a member and Chair of the $\mathrm{NCl}$ Board of Scientific Counselors Subcommittee A. She is a member of the Scientific Steering Committee and Chair of the Clinical and Translational Study Section for the Cancer Prevention \& Research Institute of Texas. She is or has been on the Scientific Advisory Boards of the Lustgarten Foundation, the Pancreatic Cancer Action Network, the V Foundation, The Alberta Canada Cancer Board, and the EORTC. She served as a member of the Oncology Drug Advisory Committee for the FDA. She has served as Deputy Director and Interim Director for the UNMC Eppley Cancer Center. She is Chief Emeritus of the Division of Medical Oncology at UCSF. She served as the founding Deputy Director and was later Director of Research Programs at the UCSF Helen Diller Family Comprehensive Cancer Center.

doi: 10.6004/jnccn.2021.0034

The ideas and viewpoints expressed in this editorial are those of the author and do not necessarily represent any policy, position, or program of NCCN. 